\title{
Defense Against Sclerotinia sclerotiorum in Arabidopsis Is Dependent on Jasmonic Acid, Salicylic Acid, and Ethylene Signaling
}

\author{
Xiaomei Guo and Henrik U. Stotz \\ Department of Horticulture, Oregon State University, Corvallis, Oregon 97330, U.S.A. \\ Submitted 3 May 2007. Accepted 18 June 2007.
}

\begin{abstract}
Genotypic differences in susceptibility of Arabidopsis thaliana to Sclerotinia sclerotiorum have not been reported due to the extreme susceptibility of this cruciferous plant. To overcome this limitation, we have established inoculation conditions that enable evaluation of differences in susceptibility to $S$. sclerotiorum among Arabidopsis mutants and ecotypes. Two coil mutant alleles conferred hypersusceptibility to $S$. sclerotiorum. The plant defensin gene PDF1.2 was no longer induced after challenging the coil-2 mutant with $S$. sclerotiorum. Hypersusceptibility of the coil-2 mutant to $S$. sclerotiorum was not correlated with oxalate sensitivity. The mutants $n p r 1$ and ein 2 were also hypersusceptible to $S$. sclerotiorum. Induction of PDF1.2 and the pathogenesis-related gene $P R 1$ was reduced in ein 2 and nprl mutants, respectively. Actigard, a commercial formulation of the systemic acquired resistance inducer benzothiadiazole, reduced susceptibility to $S$. sclerotiorum. Based on histochemical analysis of oxalate-deficient and wild-type strains of $S$. sclerotiorum, oxalate caused a decrease in hydrogen peroxide production but no detectable changes in plant superoxide production or gene expression.
\end{abstract}

Additional keywords: $\mathrm{H}_{2} \mathrm{O}_{2}$, SAR.

Protection of plants against pathogens depends on constitutive and induced defense mechanisms (Nürnberger and Lipka 2005). Various phytohormones are crucial for induced and systemic defense signaling (Lorenzo and Solano 2005). Extensive research has shown that salicylic acid (SA) plays a key role in local and systemic acquired resistance (SAR) to biotrophic pathogens (Durrant and Dong 2004; Gaffney et al. 1993). Recently, SA signaling was implicated in local resistance to the necrotrophic fungal pathogen Botrytis cinerea (Ferrari et al. 2003), but B. cinerea does not trigger SAR (Govrin and Levine 2002). Induced systemic resistance depends on jasmonic acid (JA) and ethylene (ET) signaling (Pieterse et al. 1998). Both of these hormonal signaling pathways have been shown to be particularly important for defense against necrotrophic pathogens such as B. cinerea (Thomma et al. 1998, 1999). Nevertheless, it has been acknowledged that the dichotomy of SA and JA/ET signaling for defense against biotrophic and necrotrophic pathogens, respectively, is an oversimplification (Glazebrook 2005; Thomma et al. 2001).

Sclerotinia sclerotiorum is a necrotrophic ascomycete closely related to $B$. cinerea. This destructive pathogen attacks more than 400 plant species, including Arabidopsis thaliana

Corresponding author: Henrik U. Stotz; Telephone: +1.541.737.5468; Fax: +1.541.737.3479; E-mail: stotzhe@hort.oregonstate.edu
(Boland and Hall 1994). S. sclerotiorum is distributed around the world (Purdy 1979) and causes annual crop losses exceeding \$200 million in the United States alone (Bolton et al. 2006). Despite this substantial impact on agricultural production, surprisingly little is known about the molecular mechanisms of host resistance to $S$. sclerotiorum.

Arabidopsis has been proposed as a host model to study interactions with S. sclerotiorum (Dickman and Mitra 1992). Like other host species, Arabidopsis is resistant to oxalate-deficient $S$. sclerotiorum mutants but susceptible to wild-type fungus (Godoy et al. 1990). However, the extreme susceptibility of Arabidopsis to $S$. sclerotiorum has precluded discerning genetic determinants of host resistance or susceptibility (Dai et al. 2006).

Pathological effects of oxalic acid have long been recognized (de Bary 1886). Oxalic acid acidifies infected host tissues. This action assists in destruction of host cell walls, because polygalacturonase and other hydrolytic enzymes have an acidic $\mathrm{pH}-$ optimum (Bateman and Beer 1965; Lumsden 1979). Oxalate is also a strong chelator able to remove $\mathrm{Ca}^{2+}$ ionically bound to pectin. This further enhances polygalacturonase activity, which is dependent on removal of calcium from its substrate. Oxalic acid has been shown to suppress the oxidative burst of host plants (Cessna et al. 2000), but its causal relationship to virulence remains to be determined. Moreover, deregulation of guard cells by oxalic acid is, at least partially, responsible for wilting symptoms induced by $S$. sclerotiorum (Guimaraes and Stotz 2004).

Herein, we describe an improved protocol of inoculating rosette leaves of Arabidopsis, which has made quantification of differences in susceptibility among wild-type plants and mutants with defects in JA, SA, and ET signaling possible. Interest in defense signaling was triggered by the results of a screen for oxalate sensitivity using a library of Saccharomyces cerevisiae deletion mutants. A $\Delta r i b 4$ mutant, deleted for the gene encoding lumazine synthase, was found to be one of the 19 most sensitive mutants, failing to grow at oxalic acid concentrations as low as 1 $\mu \mathrm{M}$ (Cheng et al. 2007). COS1, the Arabidopsis ortholog of RIB4, has been identified as a suppressor of a coil (coronatine insensitive) mutant, and was thus implicated in JA signaling (Xiao et al. 2004). Based on analysis of coil (Xie et al. 1998), nprl (Cao et al. 1997), and ein2 mutants (Alonso et al. 1999), the current study provides evidence for a role of all three defense pathways in defense against $S$. sclerotiorum.

\section{RESULTS}

Susceptibility of JA signaling mutants to $S$. sclerotiorum.

A new inoculation procedure was developed to detect genotypic variation in susceptibility of Arabidopsis to S. scle- 
rotiorum. It proved critical to limit nutrient availability to the fungus by using a minimal medium (Cruickshank 1983) to overcome the inherent susceptibility of Arabidopsis (Dai et al. 2006; Dickman and Mitra 1992; Guimaraes and Stotz 2004). Endo-polygalacturonase expression has been shown to be down-regulated when $S$. sclerotiorum was cultured on minimal medium relative to potato dextrose agar (PDA) (Li et al. 2004).

A significant delay in symptom development was evident when wild-type plants were inoculated with fungus grown on minimal medium relative to PDA (Fig. 1). Consequently, minimal medium was used for all of the inoculation trials with wild-type $S$. sclerotiorum reported in this study. A soft-rotting necrosis occurred as early as 1 day postinoculation. Expansion of the water-soaked lesion led to browning and collapse of the infected leaf tissue. At this stage, fungus grown on PDA consumed approximately $50 \%$ of the inoculated leaf. Differences in lesion size were measured as long as inoculated leaves were partially alive. Relative to minimal medium, in vitro cultiva-

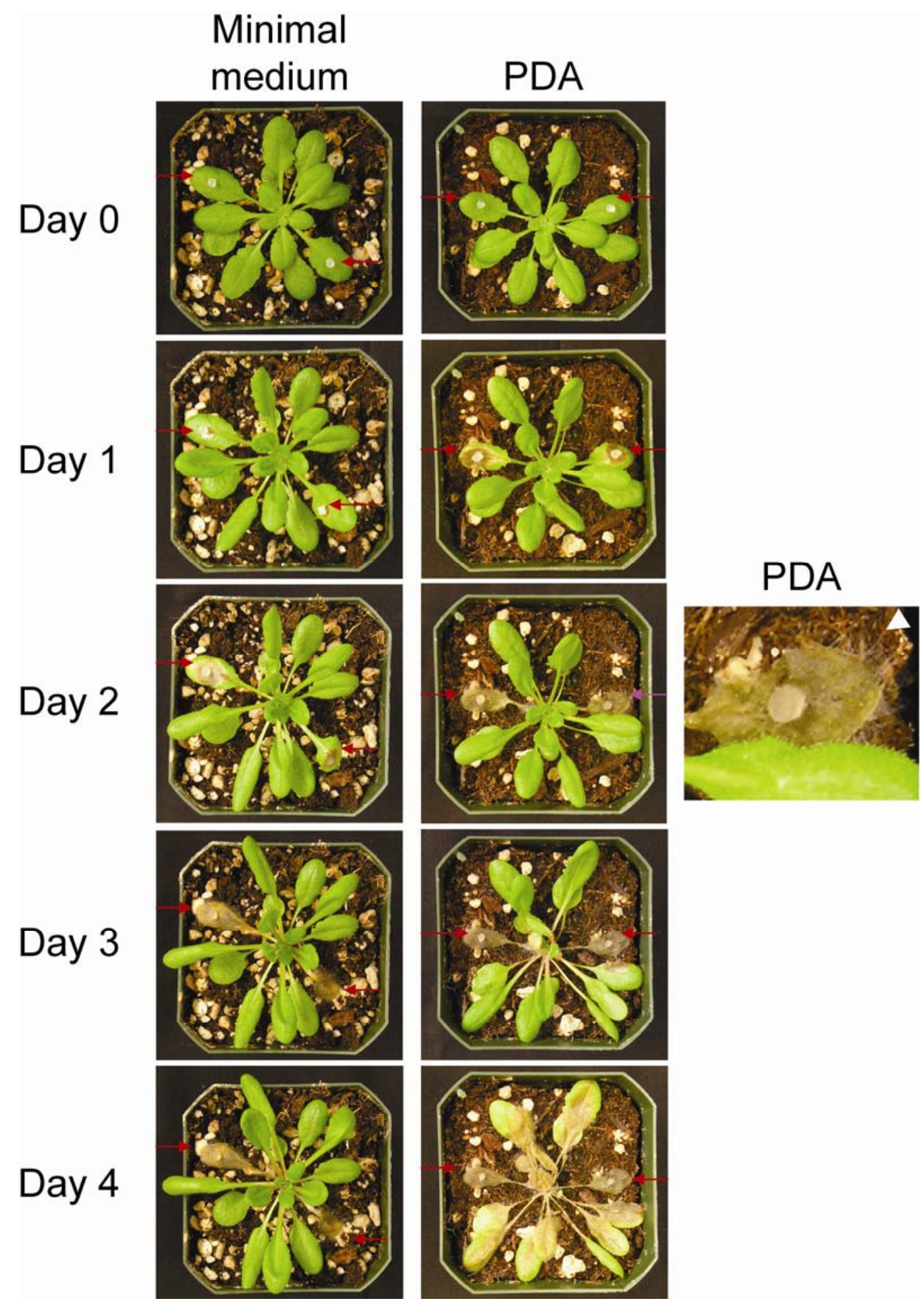

Fig. 1. Establishment of conditions to determine quantitative differences in susceptibility of Arabidopsis to Sclerotinia sclerotiorum. Nutrient-dependent differences in lesion expansion are shown. Wild-type S. sclerotiorum was cultivated on minimal medium (left column) or potato dextrose agar (PDA) prior to inoculation of Arabidopsis (Col-0). Disease progression is shown from days 0 to 4 after inoculation. Arrows indicate inoculated leaves. The collapsed leaf indicated with the pink arrow has been enlarged to visualize hyphae. The white arrowhead points towards a hypha. The width of the pots is $7.2 \mathrm{~cm}$. 
tion of $S$. sclerotiorum on PDA resulted in a 1-day-earlier consumption of inoculated leaves. Once the entire leaf was consumed, mycelia emerged from the infected tissue. As a result, lesion size could no longer be determined. Instead, the percentage of whole-plant decay was estimated.

To evaluate the possible role of JA signaling in defense against $S$. sclerotiorum and in oxalate tolerance, mutations in two different genes were analyzed. Two mutant alleles of coil, a gene encoding an F-box protein and central regulator of JA signaling (Xie et al. 1998), and jinl, a mutation in a gene encoding the downstream transcription factor AtMyc2 (Lorenzo et al. 2004), were tested.

The strong recessive coil-1 allele causes insensitivity to JA and male sterility (Feys et al. 1994), thus making propagation as heterozygous plants necessary. The coil-1 mutant has previously been shown to be hypersusceptible to $B$. cinerea and

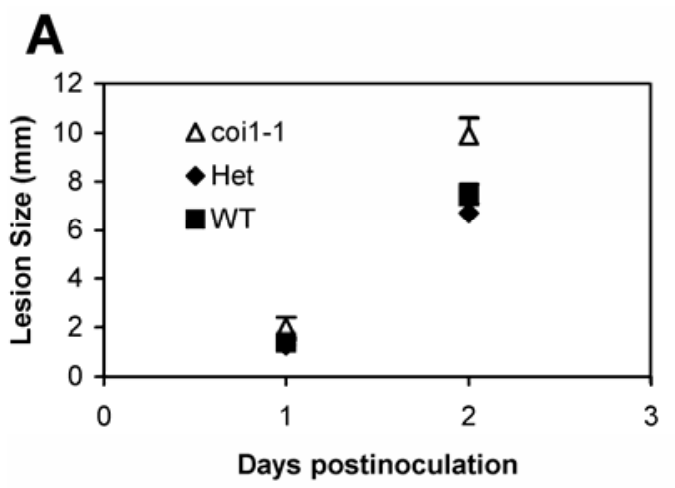

B
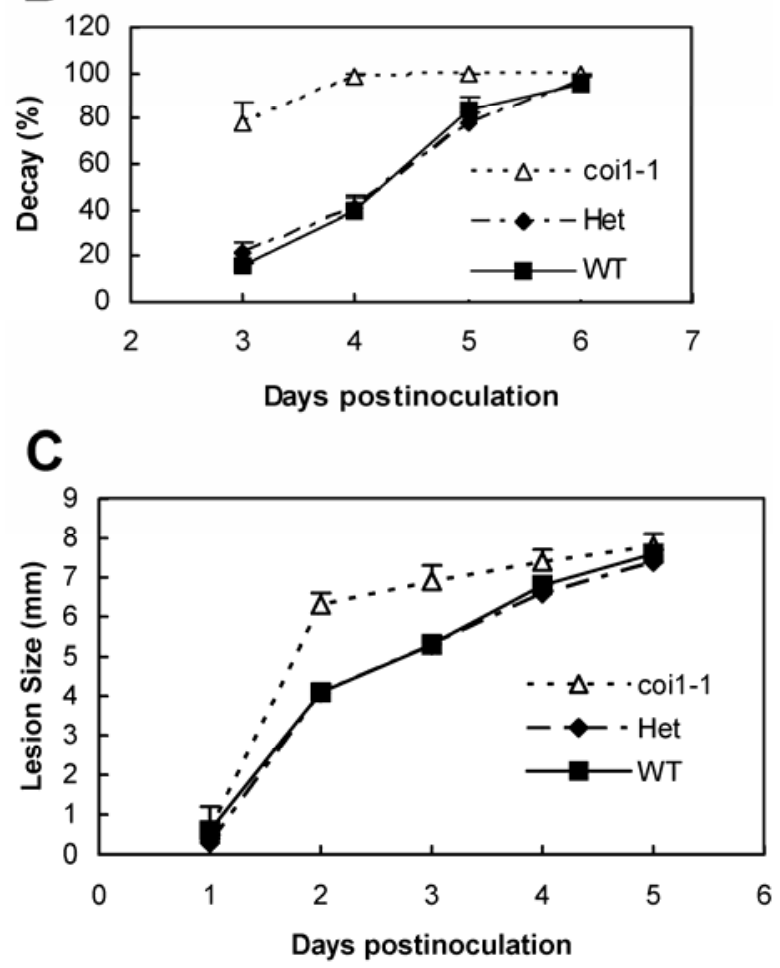

Fig. 2. Hypersusceptibility of coil-1 mutants to Sclerotinia sclerotiorum. Homozygous mutants were compared with heterozygous (Het) or wildtype (WT) plants. Means and standard errors are shown $(n>10)$. A and $\mathbf{B}$, Four- to six-week-old plants were inoculated with wild-type $S$. sclerotiorum isolate 1980 or $\mathbf{C}$, with the oxalate-deficient mutant A4. Expanding lesions (A) and (C) or whole-plant decay (B) were measured. Differences in susceptibility between coil-1 and wild-type plants were significant $(P<0.05)$ from days 2 to 4 after inoculation with wild-type $S$. sclerotiorum and from days 2 to 3 after inoculation with oxalate-deficient $S$. sclerotiorum. other necrotrophic pathogens (Thomma et al. 1998). This mutant was, therefore, a logical choice for testing the contribution of JA signaling to defense against $S$. sclerotiorum.

Progeny of heterozygous $\mathrm{COI} / \mathrm{coil}$ plants were grown. The offspring were genotyped using cleaved amplified polymorphic sequence markers (Xie et al. 1998) and were tested for susceptibility to $S$. sclerotiorum. Recessive coil-1 mutant plants were more susceptible to $S$. sclerotiorum than wild-type or heterozygous plants (Fig. 2). These differences became apparent when lesion size was measured 2 days postinoculation (Fig. 2A). Thereafter, lesions expanded beyond the inoculated leaf. Entire plants were, therefore, assessed for percentage of decay caused by the fungus to score disease development 3 days postinoculation and beyond (Fig. 2B). In contrast to the coil-1 mutant, wild-type plants did not support rapid systemic lesion expansion. The difference in rate of lesion expansion suggests that COII-mediated defenses appear to inhibit or delay the spread of the pathogen. Ultimately, however, the COI1-dependent defense pathway is overcome even in wild-type plants.

The oxalate-deficient strain A4 (Godoy et al. 1990) also accelerated lesion expansion in coil-1 mutants relative to wild-type plants. However, in plants challenged with this oxalate-deficient mutant strain, disease symptoms were limited to leaves that were inoculated and fungal spread virtually stopped after 5 days of infection (Fig. 2C). These results suggest that the coil-dependent increase in susceptibility is largely independent of oxalic acid.

In addition to coil-1, the weak coil-2 allele was tested. Homozygous coil-2 mutants are JA-insensitive but partially fertile (Xu et al. 2002) and, thus, easier to work with. These coil-2 mutants were significantly more susceptible to $S$. sclerotiorum than wild-type plants (Fig. 3A). To test JA-dependent gene ex-

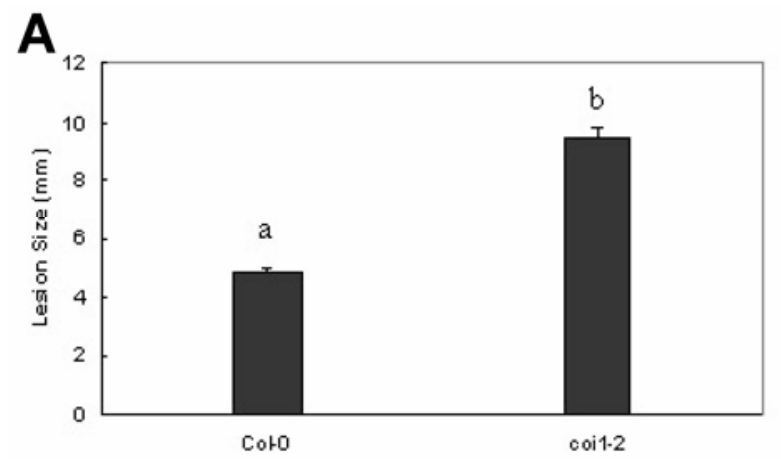

B

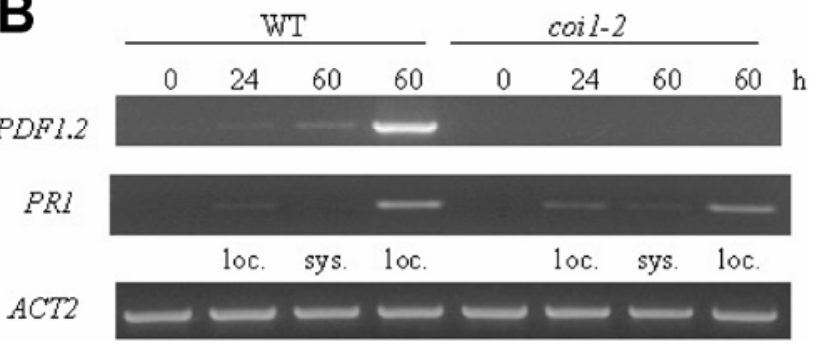

Fig. 3. Effect of jasmonic acid (JA) on defense against Sclerotinia sclerotiorum. A, Susceptibility of wild-type Col-0 $(n=63)$ and coil-2 mutant $(n=42)$ Arabidopsis to S. sclerotiorum. Lesion diameters were measured $48 \mathrm{~h}$ postinoculation. Means and standard errors are shown. Letters indicate statistically significant differences $(P<0.05)$. B, Reverse transcription-polymerase chain reaction of JA-responsive PDF1.2 (25 cycles) and salicylic acid-responsive PRI (28 cycles) after inoculation of wild-type (WT) and mutant plants with $S$. sclerotiorum; loc. = local (inoculated), sys. $=$ systemic (uninoculated) leaves. The constitutively expressed ACT2 gene ( 25 cycles) was used as a loading control. Numbers listed on the top indicate hours postinoculation. 
pression, the plant defensin gene PDF1.2 was monitored by reverse transcription-polymerase chain reaction (RT-PCR) in local and systemic leaves after inoculation of wild-type and coil-2 mutant plants with $S$. sclerotiorum (Fig. 3B). In contrast to wild-type plants, $P D F 1.2$ was not induced in pathogen-challenged coil-2 mutants. Conversely, the pathogenesis-related PRl gene was induced in both wild-type and mutant plants, illustrating that these SA-induced defense responses were not affected by the coil-2 mutation. Collectively, these data strongly suggest that the COII-dependent pathway contributes to defense against $S$. sclerotiorum.

To examine whether other mutations in the JA pathway alter susceptibility to $S$. sclerotiorum, jinl, a mutant with enhanced resistance to Fusarium oxysporum (Anderson et al. 2004) and to $B$. cinerea but with wild-type susceptibility to Alternaria brassicicola (Nickstadt et al. 2004) was tested. Seeds of the jinl mutant in the background of Col- $g l$ were obtained (Berger et al. 1996). Jasmonate insensitivity was tested by growing jinl mutants side-by-side with wild-type seedlings in the presence or absence of $50 \mu \mathrm{M}$ methyl-jasmonate (MeJA). Wildtype seedlings were more sensitive to MeJA than the jinl mutant (Fig. 4A). The coil-2 mutant was used as an additional control and was shown to be less sensitive to MeJA than the jinl mutant. For inoculation with S. sclerotiorum, seedlings were grown for 4 to 6 weeks in soil. No statistically significant differences in susceptibility to $S$. sclerotiorum were detected between jinl mutant and wild-type plants (Fig. 4B). RT-PCR analysis revealed only minor (less than twofold) differences in expression of $P D F 1.2$ and the vegetative storage protein gene VSP2 between jin1 mutants and wild-type plants (Fig. 4C). $P R 1$ was induced similarly in wild-type and mutant plants, suggesting that the SA-induced defense responses were not

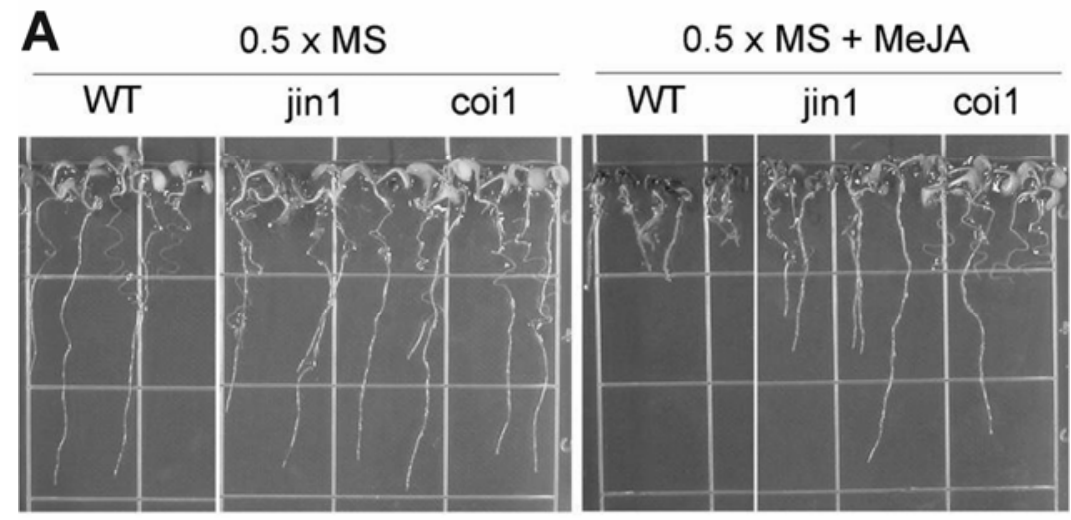

B

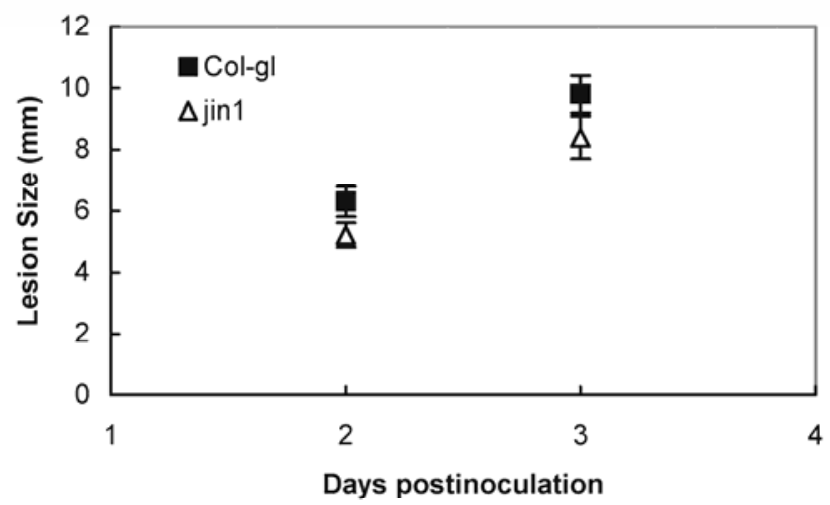

C
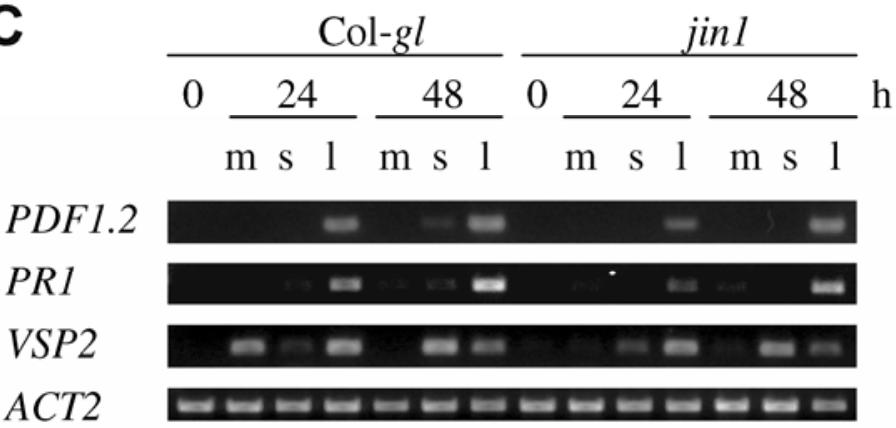

Fig. 4. Disease and defense phenotypes of the jin1 mutant. A, Confirmation of jasmonic acid sensitivity. Seedlings were grown for 1 week in the absence or presence of $50 \mu \mathrm{M}$ methyl-jasmonate (MeJA). The size of the squares is $1.35 \mathrm{~cm}$. B, Susceptibility of wild-type Col- $g l(n=33)$ and the $j i n 1$ mutant $(n=34)$ to Sclerotinia sclerotiorum. Means and standard errors are shown. C, Reverse transcription-polymerase chain reaction (RT-PCR) of PDF1.2, PR1, and VSP2 after inoculation with $S$. sclerotiorum. $\mathrm{m}=$ mock-inoculated, $\mathrm{s}=$ systemic (uninoculated), $1=$ local (inoculated) leaves. ACT2 was used as a loading control. PR1 was analyzed after 30 cycles and the other cDNAs after 25 cycles of RT-PCR. Numbers listed on the top indicated hours postinoculation. Experiments were performed twice with similar results. 
altered by the jin 1 mutation. These results demonstrate that the effects of jinl and coil mutations on susceptibility to S. sclerotiorum differ, suggesting that other transcription factors may be more important for $S$. sclerotiorum-related defense signaling than AtMyc2.
Separation of JA signaling and oxalate sensitivity.

Susceptibility to $S$. sclerotiorum is frequently (Chipps et al. 2005; Kolkman and Kelly 2000) but not always correlated with oxalate sensitivity (Kolkman and Kelly 2003). To test for oxalate sensitivity, an assay was developed by growing seedlings

A

Col-0

$\operatorname{coi} 1-2 / \cos 1$

coi1-2

MS

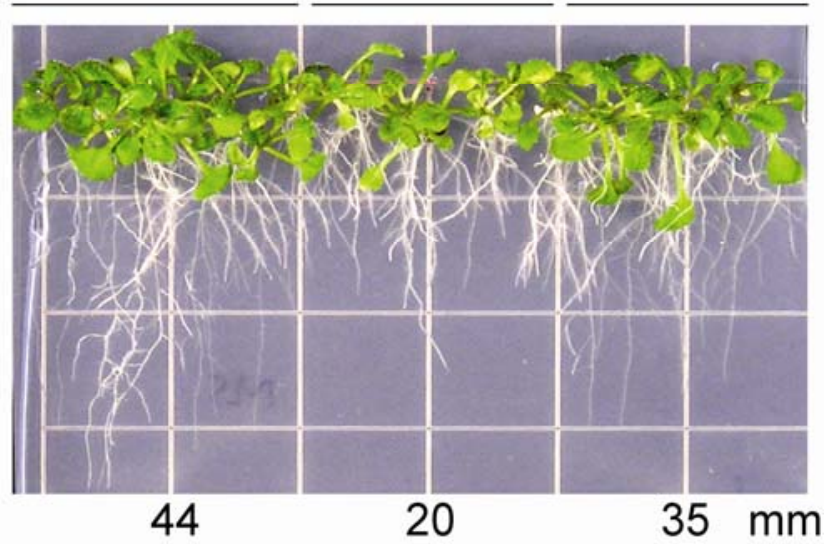

$3.5 \mathrm{mM}$ OA

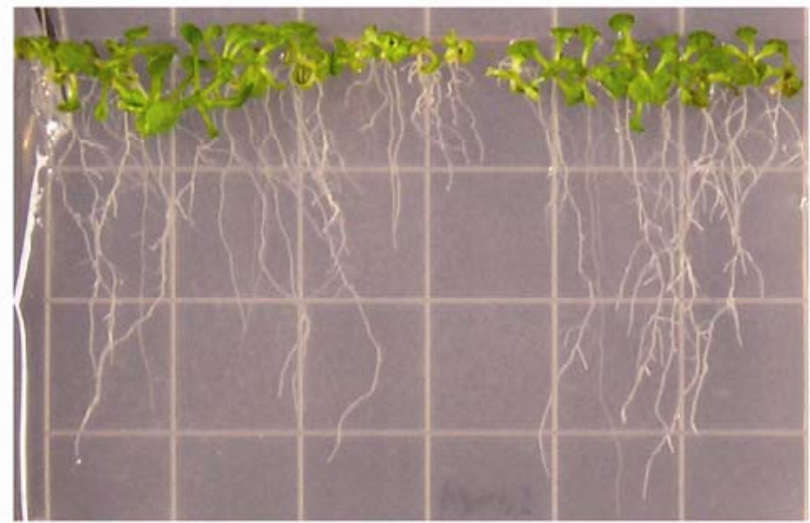

47

23

$45 \mathrm{~mm}$

$4 \mathrm{mM} O A$

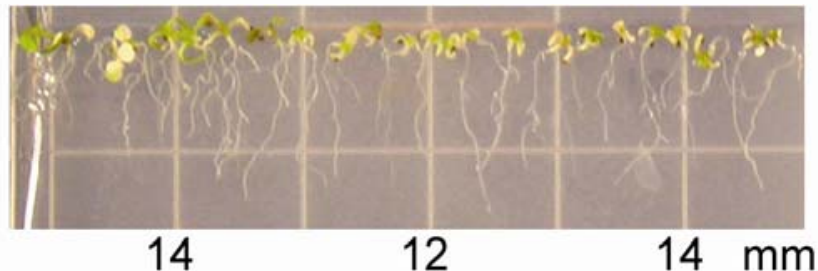

B

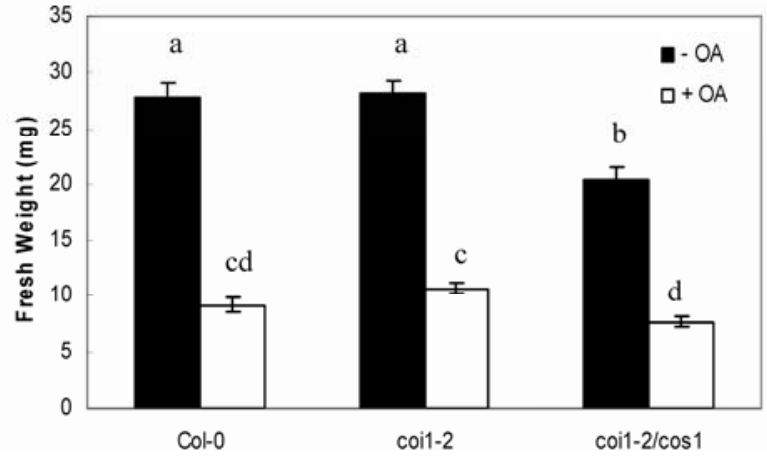

Fig. 5. Comparison of oxalate sensitivity among wild-type Col-0 plants, coil-2, and coil-2/cos 1 mutants. A, Images of seedlings grown on Murashige-Skoog (MS) medium in the absence or presence of 3.5 or $4 \mathrm{mM}$ oxalic acid (OA) for 2 weeks. Numbers beneath the images indicate average root length. The size of the squares is $1.35 \mathrm{~cm}$. B, Seedlings were grown in the absence $(-\mathrm{OA})$ or presence $(+\mathrm{OA})$ of $4 \mathrm{mM}$ oxalic acid for 3 weeks. Means and standard errors $(n \geq$ $29)$ are shown. Letters indicate statistically significant differences $(P<0.05)$. 
in Murashige-Skoog (MS) medium with or without oxalic acid at $\mathrm{pH}$ 5.5. Fresh weight or root length of Arabidopsis seedlings was measured after 2 to 3 weeks of growth.

Foliar growth and root branching was inhibited when Arabidopsis seedlings were grown on MS medium containing oxalic acid concentrations above $3 \mathrm{mM}$ (Fig. 5A). Leaf development was arrested, cotyledons became chlorotic, and root elongation was inhibited when seedlings were grown on medium containing oxalic acid concentrations above $3.5 \mathrm{mM}$. As a result, oxalic acid caused a reduction of fresh weight of wild-type plants, coil-2, and coil-2/cos 1 mutants (Fig. 5B). The double mutant was tested because deletion of RIB4, the Saccharomyces cerevisiae ortholog of COS1, increases sensitivity of budding yeast to oxalic acid (Cheng et al. 2007). The coil-2/cosl double mutant grew poorly on MS medium compared with wild-type and coil-2 mutant plants $(P<0.0001)$ when it was grown in the absence of oxalic acid. A small but significant difference was observed between coil-2/cosl and coil-2 mutants $(P=0.0298)$ when both mutants were grown in the presence of oxalic acid. Nevertheless, growth inhibition of the coil-2/cos 1 mutant relative to the $\operatorname{coi} 1-2$ mutant was 28.0 and $27.4 \%$ in the presence or absence of oxalic acid, respectively. These data show that, in contrast to yeast, lumazine synthase is likely not involved in oxalate tolerance of Arabidopsis.

Additional experiments were conducted to study doseresponse relationships by growing wild-type plants, coil-2, and coil-2/cos 1 mutant seedlings in MS medium containing 3 to $5 \mathrm{mM}$ oxalic acid at $\mathrm{pH}$ 5.5. No significant differences were observed between wild-type plants and coil-2 mutants when seedlings were grown in the presence or absence of oxalic acid (data not shown). This result demonstrates that genotypedependent differences in coil-related susceptibility to S. sclerotiorum are not caused by changes in oxalate sensitivity.

\section{Effects of oxalic acid on production \\ of reactive oxygen species (ROS) and gene expression.}

To analyze the contribution of oxalic acid to pathogenicity, wild-type Arabidopsis plants were inoculated with wild-type $S$. sclerotiorum 1980 or oxalate-deficient strain A4. The oxalatedeficient mutant was clearly less virulent than wild-type $S$. sclerotiorum (Fig. 6A), even when the former and latter strains were grown on nutrient-rich PDA and nutrient-poor minimal medium (Cruickshank 1983), respectively, thus supporting previous findings (Dickman and Mitra 1992; Guimaraes and Stotz 2004). Despite this phenotypic difference, RT-PCR analysis revealed no significant differences in temporal and spatial patterns of PDF1.2 and PRI induction when Arabidopsis was exposed to wild-type or oxalate-deficient $S$. sclerotiorum (Fig. 6B).

Staining with 3,3'-diaminobenzidine (DAB) indicated higher $\mathrm{H}_{2} \mathrm{O}_{2}$ accumulation when Arabidopsis leaves were inoculated with oxalate-deficient $S$. sclerotiorum than with wild-type fungus (Fig. 7A). This difference in DAB staining was observed when both strains were grown on minimal medium. Whereas wild-type $S$. sclerotiorum generated a spreading lesion, the oxalate-deficient mutant was confined to the area underneath the agar plug used for inoculation. Nitroblue tetrazolium (NBT)
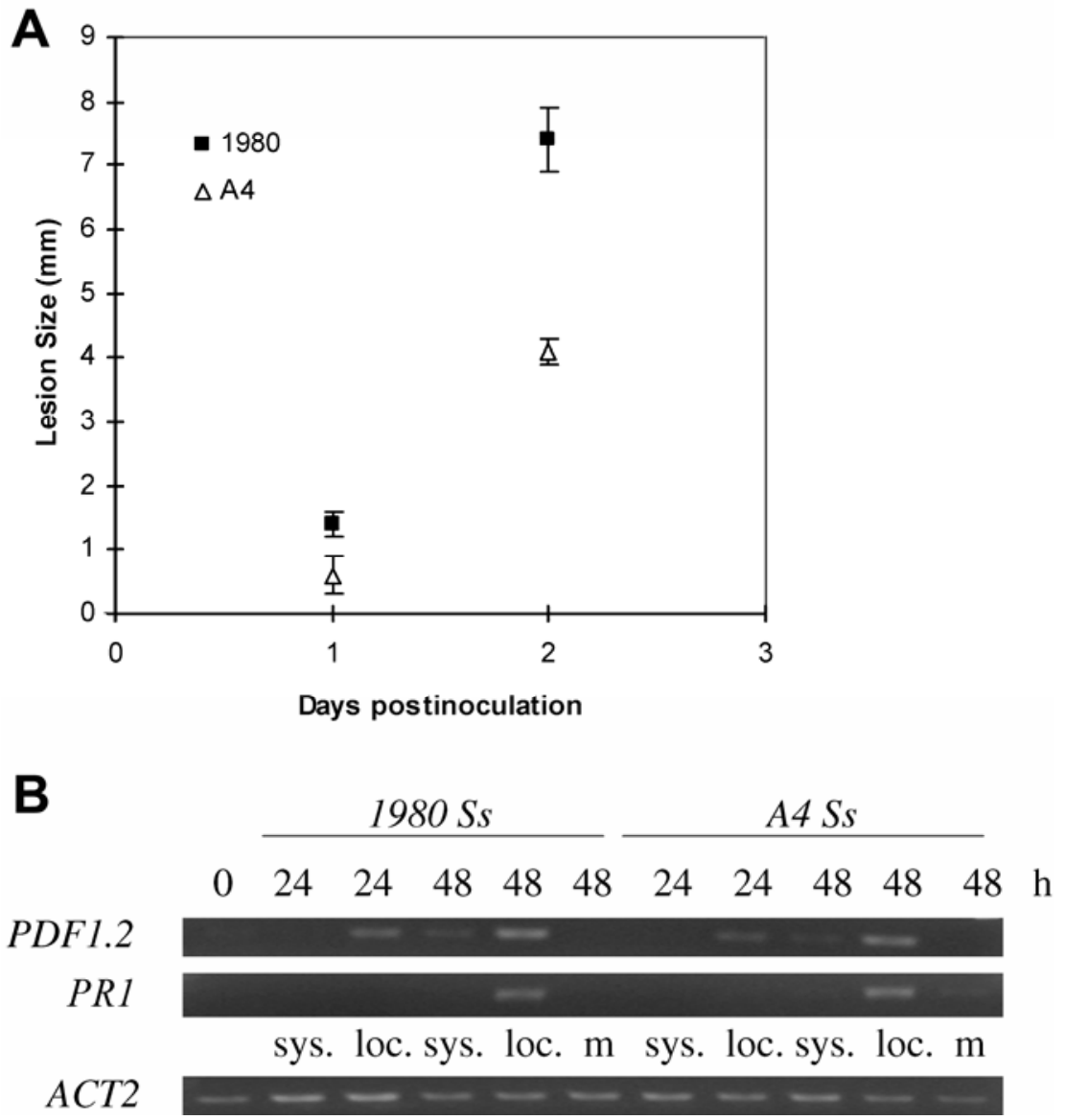

Fig. 6. Effect of oxalate on fungal virulence and plant gene expression. A, Differences in susceptibility of Arabidopsis (Col-0) to wild-type Sclerotinia sclerotiorum $1980(n=17)$ and oxalate-deficient mutant A4 $(n=21)$. Means and standard errors are shown. B, Reverse transcription-polymerase chain reaction of PDF1.2 (23 cycles) and PR1 (26 cycles) after inoculation with $S$. sclerotiorum. loc. $=$ local (inoculated), sys. $=$ systemic $($ uninoculated) leaves, $\mathrm{m}=$ mockinoculation. ACT2 (25 cycles) was used as a loading control. Numbers listed on the top indicate hours postinoculation. Wild-type and oxalate-deficient strains were grown on minimal medium and potato dextrose agar, respectively. 
staining revealed superoxide accumulation in wild-type but not in oxalate-deficient S. sclerotiorum (Fig. 7B). Superoxide accumulation was minimal in Arabidopsis leaves. Quantitative analysis suggested that DAB staining in Arabidopsis was 2.5-fold higher after inoculation with oxalate-deficient $S$. sclerotiorum relative to the wild-type strain (Fig. 7C). A 179-fold increase in NBT staining was observed in wild-type $S$. sclerotiorum relative to the oxalate-deficient fungus after inoculation of Arabidopsis. Collectively, these data suggest that oxalate enhances $\mathrm{O}_{2}{ }^{--}$production in the pathogen and suppresses $\mathrm{H}_{2} \mathrm{O}_{2}$ production in the host but has little influence on $P R I$ and PDF1.2 gene expression.

\section{Effects of SA and ET signaling on defense} against $S$. sclerotiorum.

To test whether pathways other than JA signaling are involved in resistance to $S$. sclerotiorum, susceptibilities of $n p r l$ and ein2 mutants were compared with wild-type Arabidopsis. NPRl encodes an ankyrin-repeat-containing protein that is a central regulator of SAR (Cao et al. 1994, 1997). EIN2 encodes a protein with similarities to Nramp metal-ion transporters that is a central regulator of ET signaling (Alonso et al. 1999). Comparison of ein2, coil-2, and npr1 mutants with wild-type Arabidopsis demonstrated that all three mutants are hypersusceptible to wild-type S. sclerotiorum (Fig. 8A and B). Mutations in these three pathways appear to reduce defense against $S$. sclerotiorum approximately equally. RT-PCR analysis of $P D F 1.2$ and $P R 1$ expression demonstrated that induction of these two genes by $S$. sclerotiorum was delayed and suppressed in ein 2 and npr1 mutants, respectively (Fig. 8C). Thus, S. sclerotiorum triggers SA and ET signaling in addition to JA signaling.

Similarly to the results with the JA signaling mutants, ein2 and nprl mutant seedlings grew as well as wild-type Arabidopsis when exposed to oxalate-containing medium (Fig. 8D).

\section{A DAB staining Mock}

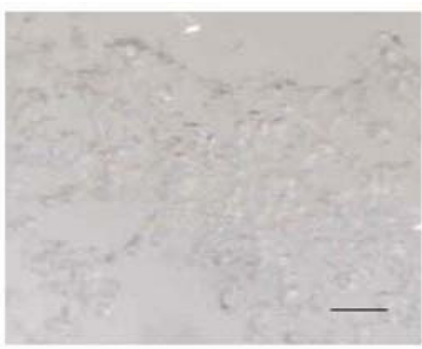

B NBT staining Mock
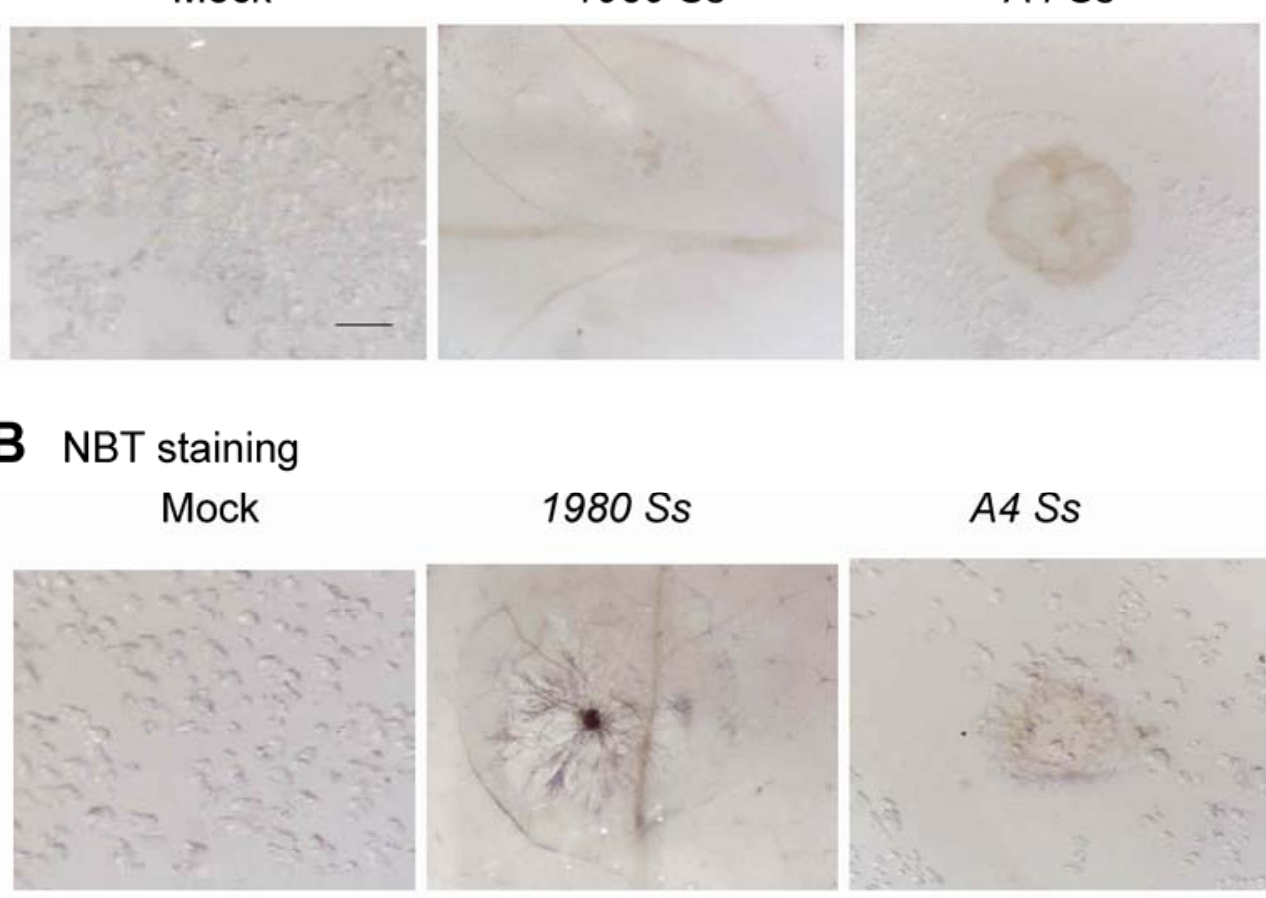

A4 Ss

C

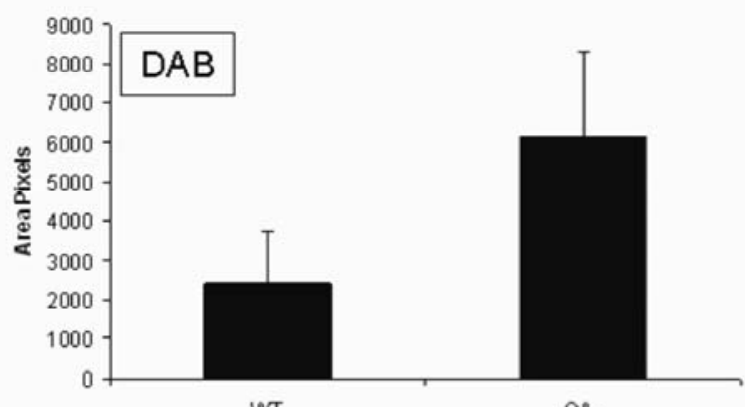

WT
A4 Ss

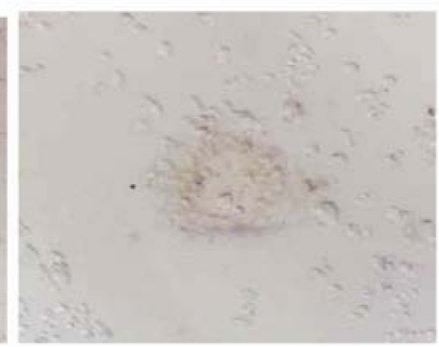

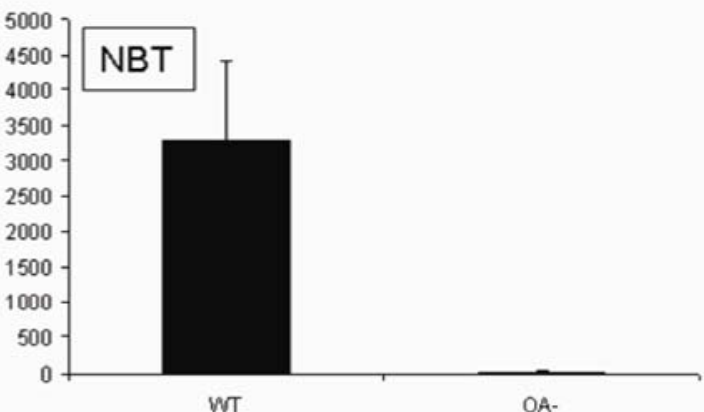

WT

Fig. 7. Effect of oxalate on reactive oxygen species (ROS) production. $\mathbf{A}, 3,3^{\prime}$-Diaminobenzidine (DAB) staining shows larger accumulation of $\mathrm{H}_{2} \mathrm{O}_{2}$ in Arabidopsis leaves inoculated with oxalate-deficient mutant Sclerotinia sclerotiorum than in leaves inoculated with wild-type S. sclerotiorum. B, Nitroblue tetrazolium (NBT) staining shows an increase in $\mathrm{O}_{2}{ }^{--}$production in wild-type strain but no major differences in ROS accumulation of Arabidopsis leaves inoculated with oxalate-deficient mutant or wild-type $S$. sclerotiorum. Mock-inoculated leaves were stained with neither DAB nor NBT. Leaf tissues were stained $48 \mathrm{~h}$ after inoculation with fungal strains grown on minimal media. The same magnification was used for all images. Enlarged portions of leaves are shown. Bar $=1 \mathrm{~mm}$. C, Quantification of DAB ( $n=4$ per strain) and NBT ( $n=3$ per strain) staining using image analysis. Differences between wild-type (WT) and oxalate-deficient (OA-) strains are shown. Means and standard errors are shown. 
A

Col-0

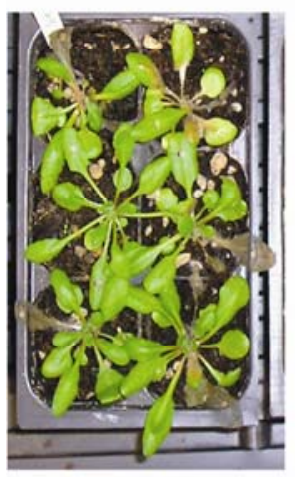

nprl

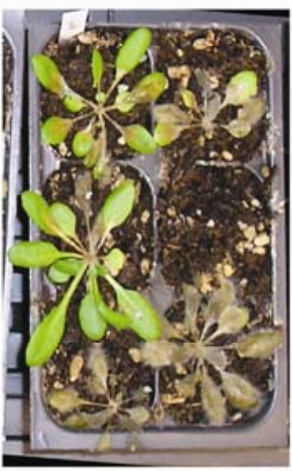

ein2

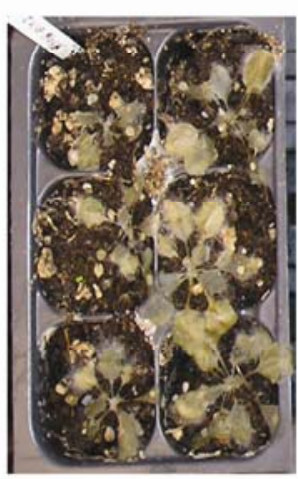

coil-2

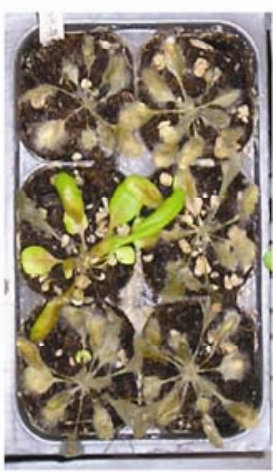

B

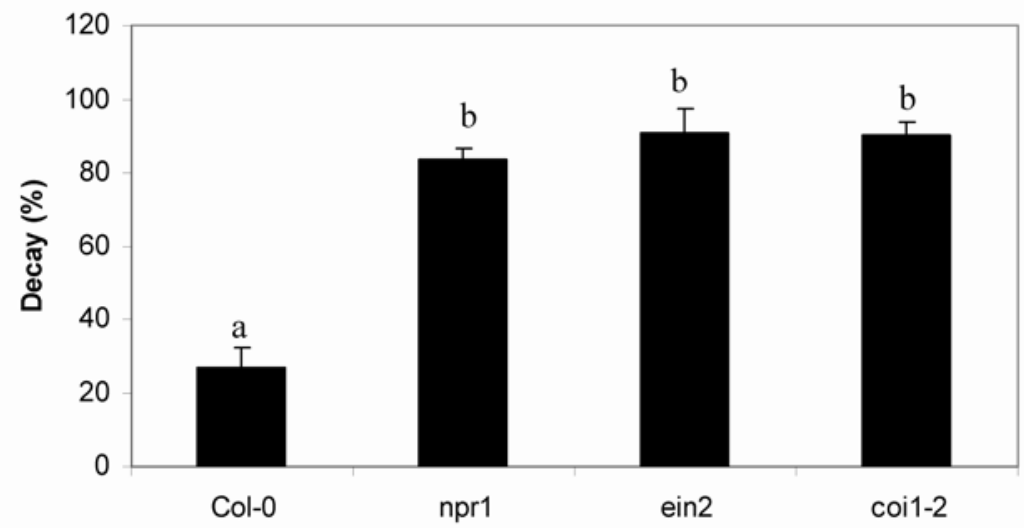

C

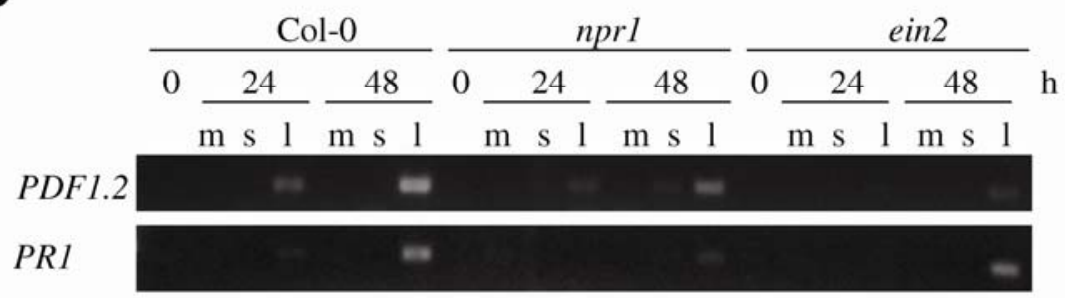

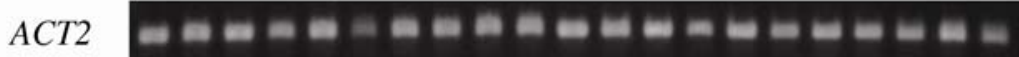

D

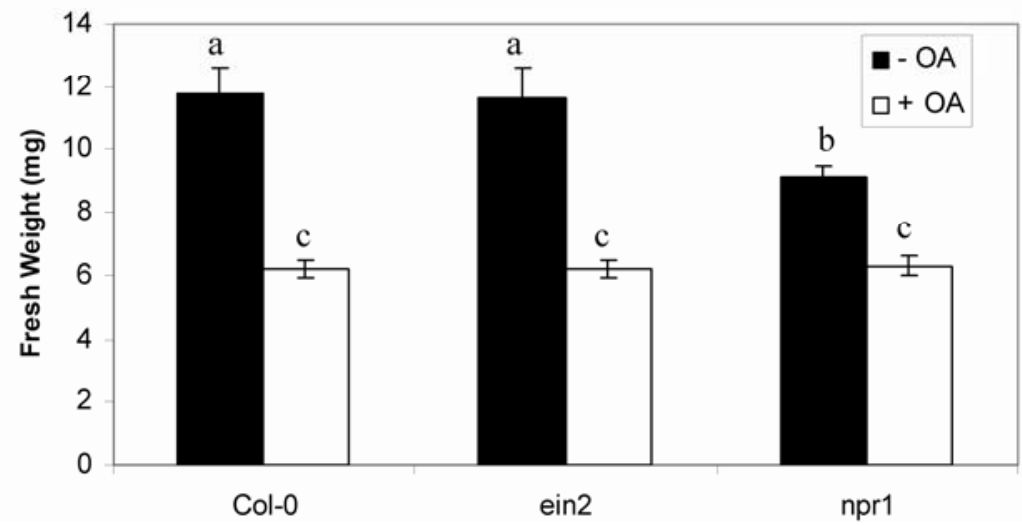

Fig. 8. Susceptibility of wild-type Col-0 plants $(n=32)$ and $n p r 1(n=51)$, ein2 $(n=10)$, and coil-2 $(n=24)$ mutants to wild-type Sclerotinia sclerotiorum. A, Photograph of inoculated plants 5 days postinoculation with the S. sclerotiorum isolate 1980. B, Quantitative analysis of whole plant decay. Means and standard errors are shown. Letters indicate statistically significant differences $(P<0.05)$. C, Reverse transcription-polymerase chain reaction of $P D F 1.2(25$ cycles) and $P R 1$ ( 28 cycles) after inoculation with $S$. sclerotiorum. $\mathrm{m}=$ mock-inoculated, $\mathrm{s}=$ systemic (uninoculated), $1=$ local (inoculated) leaves. ACT2 ( 25 cycles) was used as a loading control. Numbers listed on the top indicate hours postinoculation. D, Seedlings were grown in the absence (-OA) or presence $(+\mathrm{OA})$ of $3.5 \mathrm{mM}$ oxalic acid for 2 weeks. Means and standard errors $(n \geq 22)$ are shown. Letters indicate statistically significant differences $(P<0.05)$. 
However, a significant reduction of growth was observed when nprl mutants were grown in the absence of oxalic acid. These results suggest that none of these three signaling pathways are influenced by oxalic acid.

Hypersusceptibility of $n p r l$ mutants to S. sclerotiorum suggested that SA is involved in defense against $S$. sclerotiorum. To test whether chemical activators of SAR can be used to enhance resistance against this fungal pathogen, benzothiadiazole (BTH) was used (Lawton et al. 1996; Ward et al. 1991). The ecotypes Col-0 and Ws-0 were sprayed with BTH 4 days prior to inoculation with $S$. sclerotiorum. Resistance of Col-0 and Ws-0 to $S$. sclerotiorum was significantly increased by treatment with BTH (Fig. 9). As expected, BTH did not have an effect on nprl mutants because this chemical acts upstream of NPR1 (Shah et al. 1997, 1999).

\section{DISCUSSION}

The results of this study clearly implicate JA, SA, and ET in activation of defense responses against $S$. sclerotiorum. The coil, npr1, and ein2 mutants were all hypersusceptible to $S$. sclerotiorum. Moreover, signaling through these pathways was demonstrated using the well-characterized marker genes $P D F 1.2$ and PR1. It was previously suggested that the SA pathway protects against biotrophic pathogens, whereas JA and ET signaling enhances resistance to necrotrophic pathogens (Thomma et al. 1998). However, our study clearly shows that all three pathways are involved in defense against $S$. sclerotiorum. Similar to our observation, local resistance to $B$. cinerea is enhanced by the SA pathway, although nprl mutants were as susceptible as wild-type plants (Ferrari et al. 2003). The nprl mutation only increases susceptibility to $B$. cinerea in the background of the ein2 mutation (Ferrari et al. 2003). Thus, our study on S. sclerotiorum adds to recent conclusions that strict separation of defenses against nectrotrophic and biotrophic pathogens via SA and JA/ET signaling, respectively, is an oversimplification (Glazebrook 2005; Thomma et al. 2001). The rapid death of pathogen-infected nprl, coil, and ein2 mutants suggests that induced and systemic resistance slows the progression of $S$. sclerotiorum in wild-type plants.

Three lines of evidence suggest that oxalic acid does not influence JA-induced partial resistance to S. sclerotiorum. First, coil-1 mutants are hypersusceptible to both wild-type and oxalate-deficient $S$. sclerotiorum. Second, hypersusceptibility of the coil-2 mutant to $S$. sclerotiorum does not correlate with changes in oxalate sensitivity. Third, despite differences in virulence between wild-type and oxalate-deficient $S$. scle- rotiorum, no oxalate-dependent differences in JA-related gene expression were observed. These findings strongly suggest that oxalic acid does not influence JA signaling.

Host resistance mechanisms to $S$. sclerotiorum have not received much attention. Conversely, heterologous overexpression of oxalate oxidase or oxalate decarboxylase genes has previously been used to protect dicots against $S$. sclerotiorum (Donaldson et al. 2001; Kesarwani et al. 2000). Oxalate oxidases and oxalate decarboxylase are derived from cereals (Lane et al. 1993), which are not hosts of S. sclerotiorum, and the microbe Collybia velutipes (Mehta and Datta 1991), respectively.

In addition to the coil mutants, the jinl mutant was used to analyze JA signaling. Susceptibilities of jinl mutant and wildtype plants to $S$. sclerotiorum were not significantly different. This result suggests that transcription factors other than JIN1, like ERF1, are involved in protecting wild-type plants against $S$. sclerotiorum (Anderson et al. 2004). Overexpression of ERF1 increases resistance to several necrotrophic fungi (Berrocal-Lobo et al. 2002), whereas JIN1 appears to be a negative regulator of plant defense responses (Lorenzo et al. 2004). These findings imply antagonistic interaction between ERF1 and JIN1. A JA-insensitive mutant would be expected to lower the expression of JA-pathway genes. However, our RTPCR results showed no significant genotype-dependent changes in $P D F 1.2$ and $V S P 2$ expression in jin1 mutants relative to wild-type plants. PDF1.2 expression is down-regulated in jinl mutants relative to wild-type plants after inoculation with B. cinerea (Nickstadt et al. 2004). On the contrary, $P D F 1.2$ expression is up-regulated in jinl mutants exposed to virulent Pseudomonas syringae (Laurie-Berry et al. 2006). Thus, regulation of PDF1.2 expression by JIN1 varies among different plant-pathogen interactions. It is possible that the absence of altered PDF1.2 and PRI expression in jinl mutants challenged with Sclerotinia sclerotiorum is due to simultaneous activation of JA and SA signaling.

JA-induced but not wound-induced expression of VSP is suppressed in leaves of jinl mutants relative to wild-type plants (Berger et al. 1996). These results are compatible with our observation that jinl mutants do not differ from wild-type plants in terms of VSP2 induction after inoculation with $S$. sclerotiorum. It was previously suggested that JIN1 differentially regulates two branches of the JA pathway (Lorenzo et al. 2004). Specifically, JIN1 was proposed to activate the wound response while repressing pathogen responses. These conclusions were based on downregulation of VSP2 expression and upregulation of $P D F 1.2$ expression in jin1 mutants relative to wild-type plants after treatment with JA. Collectively, these

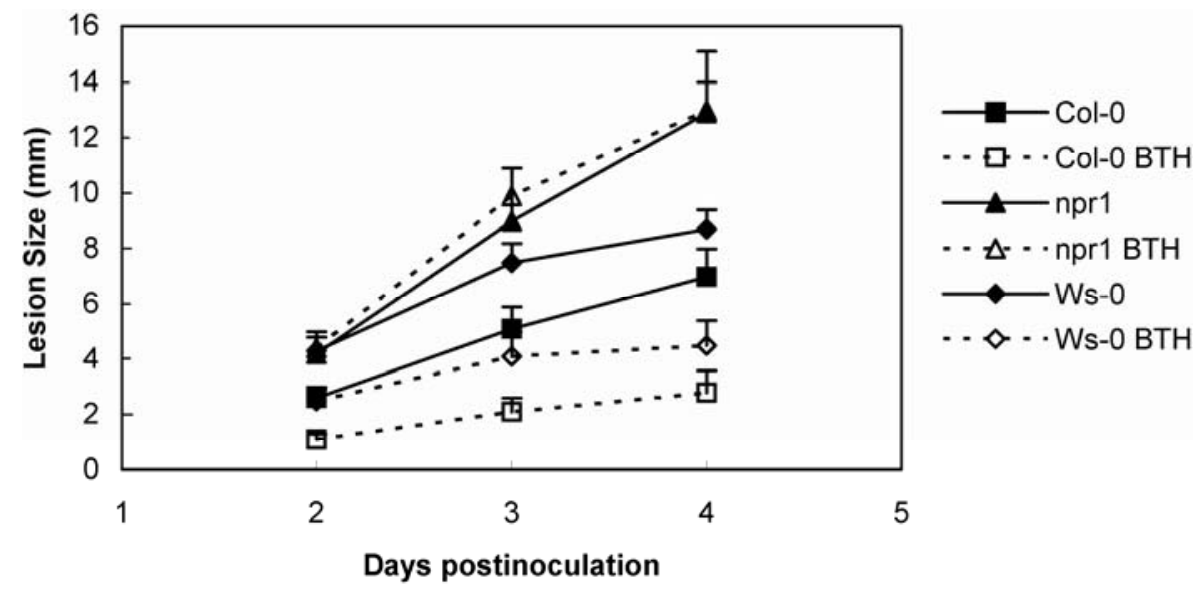

Fig. 9. Effect of benzothiadiazole $(\mathrm{BTH})$ on resistance of Arabidopsis to wild-type Sclerotinia sclerotiorum in ecotypes Col-0 $(n=18)$ and Ws- 0 ( $n=18)$ but not in $n \operatorname{prl}(n=6)$ mutants. Means and standard errors are shown. Plants were sprayed with water or $300 \mu \mathrm{M}$ BTH 4 days prior to inoculation. 
data suggest that JA and pathogens differentially regulate $P D F 1.2$ and VSP2 gene expression in jin1 mutants (Lorenzo et al. 2004; Nickstadt et al. 2004).

We analyzed the relationship between oxalate sensitivity and JA signaling because the Saccharomyces cerevisiae mutant $\Delta r i b 4$ was shown to be hypersensitive to oxalic acid (Cheng et al. 2007). However, the cos 1 mutation of the orthologous gene did not increase oxalate sensitivity in Arabidopsis. Lumazine synthases encoded by RIB4 and COS1 are pentamers and 60meric icosahedral capsids in fungi and plants, respectively (Persson et al. 1999). In addition, lumazine synthase is targeted to the chloroplast in plants (Jordan et al. 1999), whereas the enzyme in budding yeast has been localized to the cytoplasm and to the nucleus (Huh et al. 2003). Differences in protein structure and cellular localization may therefore be responsible for the different phenotypic effects of fungal and plant lumazine synthases.

We confirmed earlier findings that oxalate-deficient mutants of $S$. sclerotiorum are less virulent than wild-type fungus in Arabidopsis (Dickman and Mitra 1992). It was previously shown that oxalic acid suppresses the oxidative burst of host plants (Cessna et al. 2000). Accordingly, DAB staining indicated that $\mathrm{H}_{2} \mathrm{O}_{2}$ accumulation increased when wild-type Arabidopsis plants inoculated with oxalate-deficient $S$. sclerotiorum relative to wild-type fungus. However, we could not discern any oxalate-dependent suppression of NBT staining indicative of $\mathrm{O}_{2}{ }^{--}$production in tobacco (data not shown) or Arabidopsis. Unlike Cessna and associates (2000), we used the oxalate-deficient mutant strain A4, which could imply strain-specific differences in elicitation of ROS. We also observed no significant changes in PRI expression after inoculation of Arabidopsis with oxalate-deficient or wild-type $S$. sclerotiorum, although oxalate and $\mathrm{H}_{2} \mathrm{O}_{2}$ are expected to change SA-dependent gene expression ( $\mathrm{Hu}$ et al. 2003). Oxalate-dependent differences in DAB staining were only observed after inoculation of Arabidopsis with $S$. sclerotiorum precultured on minimal medium. PRI expression was not up-regulated after inoculation of the host with oxalate-deficient fungus pregrown on PDA (Fig. 6B) or minimal medium (data not shown). Possibly, the amount of $\mathrm{H}_{2} \mathrm{O}_{2}$ production triggered by the oxalate-deficient strain was insufficient to significantly increase $P R l$ expression. Effects of oxalate on pectin metabolism may, therefore, be as or even more important than the suppression of induced host defenses (Bateman and Beer 1965).

In addition to testing the involvement of SA signaling genetically, we determined the effects of BTH on resistance to $S$. sclerotiorum. Similarly to a previous study in soybean (Dann et al. 1998), we showed that BTH significantly increased resistance of wild-type Arabidopsis to S. sclerotiorum. Thus, activation of SAR may be used commercially to improve resistance of crop species to $S$. sclerotiorum in the field.

\section{MATERIALS AND METHODS}

\section{Biological material and growth conditions.}

Homozygous coil-2 and coil-2/cosl mutants were generously provided by D. Xie (National University of Singapore, Republic of Singapore). The jinl mutant and Col-gl wild-type seeds were donated by S. Berger (Bayerische Julius-Maximilians-Universität, Würzburg, Germany). The coil-1 mutant seeds came from J. G. Turner (University of East Anglia, Norwich, U.K.). All other lines were obtained from the Arabidopsis Biological Resource Center. A randomized complete block design was used to grow plants in 72-cell flats. Arabidopsis thaliana seeds were sown on wet Sunshine SB40 soil mix (Sun Gro Horticulture, Bellevue, WA, U.S.A.) and were stratified at $4^{\circ} \mathrm{C}$ in the dark for up to 5 days. An MB-60B growth chamber
(Percival Scientific, Perry, IA, U.S.A.) was used for plant cultivation. A light intensity of $300 \mu \mathrm{mol} \mathrm{m} \mathrm{m}^{-2} \mathrm{~s}^{-1}$ and a photoperiod of $11 \mathrm{~h}$ of light and $13 \mathrm{~h}$ of darkness were used at $22^{\circ} \mathrm{C}$. Fungal strains have previously been obtained from M. Dickman (Guimaraes and Stotz 2004).

\section{Chemical treatment.}

Each soil-grown plant was sprayed with approximately 0.6 $\mathrm{ml}$ of $300 \mu \mathrm{M}$ BTH in the form of Actigard 50WG (Syngenta, Greensboro, NC, U.S.A.) or water. Plants were dried under ambient laboratory conditions for $2 \mathrm{~h}$ and, then, were moved back to the growth chamber. Plants were inoculated with $S$. sclerotiorum 4 days later.

\section{Plant inoculation.}

Oxalate-deficient S. sclerotiorum A4 (Godoy et al. 1990) was cultured on PDA or on minimal medium $(1 \mathrm{~g}$ of $\mathrm{NaOH}, 3$ $\mathrm{g}$ of DL-malic acid, $2 \mathrm{~g}$ of $\mathrm{NH}_{4} \mathrm{NO}_{3}, 0.1 \mathrm{~g}$ of $\mathrm{MgSO}_{4} \times 7 \mathrm{H}_{2} \mathrm{O}$, and $39 \mathrm{~g}$ of Bacto-agar per liter) to reduce the aggressiveness of the fungus (Cruickshank 1983). Wild-type S. sclerotiorum isolate 1980 was cultured exclusively on minimal medium prior to inoculation. An agar plug ( $2.5 \mathrm{~mm}$ in diameter) containing the advancing edge of $S$. sclerotiorum mycelia was removed to inoculate Arabidopsis leaves. A single rosette leaf of 4- to 6-week-old Arabidopsis plants was inoculated for gene expression data. Two rosette leaves per plant were inoculated for phenotypic experiments. Lengths and widths of lesions were measured with a caliper before disease symptoms expanded beyond inoculated leaves. Decay percentage of individual plants was scored after lesions expanded beyond the inoculated leaf to assess disease development.

\section{RT-PCR analysis.}

Inoculated and uninoculated leaves were collected at various stages after infection with $S$. sclerotiorum. Total RNA was extracted from Arabidopsis leaves using the phenol-LiCl method (Sambrook et al. 1989). cDNA was synthesized using $2 \mu \mathrm{g}$ of RNA from each treatment and a first-strand synthesis kit (GE Healthcare, Piscataway, NJ, U.S.A.). PCR was run from 23 to 30 cycles with primers for PDF1.2, VSP2, PR1, and actin2 at annealing temperatures of $67,57,51$, and $55^{\circ} \mathrm{C}$, respectively. Primers for amplification of $P D F 1.2$ were 5'-AATGAGCTCT CATGGCTAAGTTTGCTTCC-3' and 5'-AATCCATGGAATA CACACGATTTAGCACC-3'. Primers for amplification of VSP2 were 5'-GTACTGGTTGTGGTTAGGGAC-3' and 5'-GAAGG TACGTAGTAGAGTGG-3'. Primers for amplification of $P R I$ were 5'-CTCAAGATAGCCCACAAGA-3' and 5'-TAGTATGG CTTCTCGTTCAC-3'. Primers for amplification of actin2 were 5'-CAGAGCGGGAAATTGTAAGAGAC-3' and 5'-ACAAAA AGGGAAATGAAACAAACA-3'.

\section{Oxalate sensitivity assay.}

Square petri dishes $(100 \times 15 \mathrm{~mm})$ were used to grow plants vertically. MS basal medium with sucrose and agar (Sigma, St. Louis) was used as a control. To measure oxalate sensitivity, MS medium was supplemented with 3 to $5 \mathrm{mM}$ oxalic acid at $\mathrm{pH}$ 5.5. Seeds were surface-sterilized, and 10 seeds of each genotype (Col-0, coil-2, and coil-2/cosl) were placed sideby-side on each plate. Growth was determined by measuring root length or fresh weight of individual seedlings after 2 to 3 weeks of growth.

\section{NBT staining.}

Leaves were detached 24 and $48 \mathrm{~h}$ postinoculation and were vacuum-infiltrated in $2 \mathrm{ml}$ of $10 \mathrm{mM}$ potassium phosphate buffer, $\mathrm{pH} 7.5$, containing $10 \mathrm{mM} \mathrm{NaN}_{3}$ and $0.1 \% \mathrm{NBT}$ (Sigma) for $30 \mathrm{~min}$ at room temperature (Sang et al. 2001). 
Leaves were boiled in $96 \%$ ethanol for 10 min before visualizing blue precipitates. ImageJ software (National Institutes of Health, Bethesda, MD, U.S.A.) was used to quantify the intensity of staining. A threshold of 0 to 150 was used, the minimal particle size was set to 50, and the same region of interest was used for all images.

\section{$\mathrm{H}_{2} \mathrm{O}_{2}$ detection by DAB staining.}

Leaves were detached $48 \mathrm{~h}$ postinoculation. Individual leaf petioles were dipped into $1 \mathrm{mg}$ of DAB per milliliter (Sigma), $\mathrm{pH} 3.8$, and were incubated for $8 \mathrm{~h}$ prior to the end of the experiment in the dark at room temperature. Thereafter, leaves were removed, placed into acetic acid/glycerol/ethanol (1:1:1, $\mathrm{vol} / \mathrm{vol} / \mathrm{vol}$ ) and were boiled for $5 \mathrm{~min}$ in water bath. Subsequently, the solution was exchanged and leaves were maintained in $60 \%$ glycerol. $\mathrm{H}_{2} \mathrm{O}_{2}$ was detected as a brown precipitate in inoculated leaves, using a microscope (Thordal-Christensen et al. 1997). ImageJ software (National Institutes of Health) was used to quantify the intensity of staining. A threshold of 0 to 175 was used, the minimal particle size was set to 50 , and the same region of interest was used for all images.

\section{Statistical analysis.}

The SAS program package (Cary, NC, U.S.A.) was used for statistical analysis. Specifically, the PROC general linear model was used to analyze disease or oxalate sensitivity data. LSMEANS and PDIFF were used to separate significant differences among means that were explained by genotype, treatment, or genotype-by-treatment interaction. Levene's test was used to ascertain homogeneity of variances.

\section{ACKNOWLEDGMENTS}

We thank D. Xie for supply of coil-2 and coil-2/cos 1 mutants and S Berger for the jin1 mutant. We are grateful to L. Buchwaldt (Agriculture and Agri-Food, Canada), who recommended the use of the Cruickshank minimal medium. We appreciate the contributions of L. Meek and S. Viner to the establishment of oxalate sensitivity assays. We also appreciate the critical reviews of this manuscript by M. Mok, J. Myers, and A. Bakalinsky. This research was funded by grant number 58-5442-4-282 from the U.S. Department of Agriculture National Sclerotinia Initiative Special Grants program.

\section{LITERATURE CITED}

Alonso J. M., Hirayama, T., Roman, G., Nourizadeh, S., and Ecker, J. R. 1999. EIN2, a bifunctional transducer of ethylene and stress responses in Arabidopsis. Science 284:2148-2152.

Anderson J. P., Badruzsaufari, E., Schenk, P. M., Manners, J. M., Desmond, O. J., Ehlert, C., Maclean, D. J., Ebert, P. R., and Kazan, K. 2004. Antagonistic interaction between abscisic acid and jasmonate-ethylene signaling pathways modulates defense gene expression and disease resistance in Arabidopsis. Plant Cell 16:3460-3479.

Bateman D. F., and Beer, S. V. 1965. Simultaneous production and synergistic action of oxalic acid and polygalacturonase during pathogenesis of Sclerotium rolfsii. Phytopathology 55:204-211.

Berger S., Bell, E., and Mullet, J. E. 1996. Two methyl jasmonate-insensitive mutants show altered expression of AtVsp in response to methyl jasmonate and wounding. Plant Physiol. 111:525-531.

Berrocal-Lobo M., Molina, A., and Solano, R. 2002. Constitutive expression of ETHYLENE-RESPONSE-FACTOR1 in Arabidopsis confers resistance to several necrotrophic fungi. Plant J. 29:23-32.

Boland G. J., and Hall, R. 1994. Index of plant hosts of Sclerotinia sclerotiorum. Can. J. Plant Pathol. 16:93-108.

Bolton M. D., Thomma, B. P. H. J., and Nelson, B. D. 2006. Sclerotinia sclerotiorum (Lib.) de Bary: Biology and molecular traits of a cosmopolitan pathogen. Mol. Plant Pathol. 7:1-16.

Cao H., Bowling, S. A., Gordon, A. S., and Dong, X. 1994. Characterization of an Arabidopsis mutant that is nonresponsive to inducers of systemic acquired resistance. Plant Cell 6:1583-1592.

Cao H., Glazebrook, J., Clarke, J. D., Volko, S., and Dong, X. 1997. The Arabidopsis NPR1 gene that controls systemic acquired resistance encodes a novel protein containing ankyrin repeats. Cell 88:57-63.
Cessna S. G., Sears, V. E., Dickman, M. B., and Low, P. S. 2000. Oxalic acid, a pathogenicity factor for Sclerotinia sclerotiorum, suppresses the oxidative burst of the host plant. Plant Cell 12:2191-2199.

Cheng V., Stotz, H. U., Hippchen, K., and Bakalinsky, A. T. 2007. Genome-wide screen for oxalate-sensitive mutants of Saccharomyces cerevisiae. Appl. Environ. Microbiol. 73:5919-5927.

Chipps T. J., Gilmore, B., Myers, J. R., and Stotz, H. U. 2005. Relationship between oxalate, oxalate oxidase activity, oxalate sensitivity, and white mold susceptibility in Phaseolus coccineus. Phytopathology 95:292-299.

Cruickshank R. H. 1983. Distinction between Sclerotinia species by their pectic zymograms. Trans. Brit. Mycol. Soc. 80:117-119.

Dai F.-M., Xu, T., Wolf, G. A., and He, Z.-H. 2006. Physiological and molecular features of the pathosystem Arabidopsis thaliana L.-Sclerotinia sclerotiorum Libert. J. Integr. Plant Biol. 48:44-52.

Dann E., Diers, B., Byrum, J., and Hammerschmidt, R. 1998. Effect of treating soybean with 2,6-dichloroisonicotinic acid (INA) and benzothiadiazole (BTH) on seed yields and the level of disease caused by Sclerotinia sclerotiorum in field and greenhouse studies. Eur. J. Plant Pathol. 104:271-278.

de Bary A. 1886. Ueber einige Sclerotinien and Sclerotienkrankheiten. Botanische Zeitung 44:377-474.

Dickman M. B., and Mitra, A. 1992. Arabidopsis thaliana as a model for studying Sclerotinia sclerotiorum pathogenesis. Physiol. Mol. Plant Pathol. 41:255-263.

Donaldson P. A., Anderson, T., Lane, B. G., Davidson, A. L., and Simmonds, D. H. 2001. Soybean plants expressing an active oligomeric oxalate oxidase from the wheat gf-2.8 (germin) gene are resistant to the oxalate-secreting pathogen Sclerotinia sclerotiorum. Physiol. Mol. Plant Pathol. 59:297-307.

Durrant W. E., and Dong, X. 2004. Systemic acquired resistance. Annu. Rev. Phytopathol. 42:185-209.

Ferrari S., Plotnikova, J. M., De, L. G., and Ausubel, F. M. 2003. Arabidopsis local resistance to Botrytis cinerea involves salicylic acid and camalexin and requires EDS4 and PAD2, but not SID2, EDS5 or PAD4. Plant J. 35:193-205.

Feys B. J. F., Benedetti, C. E., Penfold, C. N., and Turner, J. G. 1994 Arabidopsis mutants selected for resistance to the phytotoxin coronatine are male sterile, insensitive to methyl jasmonate, and resistant to a bacterial pathogen. Plant Cell 6:751-759.

Gaffney T., Friedrich, L., Vernooij, B., Negrotto, D., Nye, G., Uknes, S., Ward, E., Kessmann, H., and Ryals, J. 1993. Requirement of salicylic acid for the induction of systemic acquired resistance. Science 261:754756.

Glazebrook J. 2005. Contrasting mechanisms of defense against biotrophic and necrotrophic pathogens. Annu. Rev. Phytopathol. 43:205-227.

Godoy G., Steadman, J. R., Dickman, M. B., and Dam, R. 1990. Use of mutants to demonstrate the role of oxalic acid in pathogenicity of Sclerotinia sclerotiorum on Phaseolus vulgaris. Physiol. Mol. Plant Pathol. 37:179-191.

Govrin E., M, and Levine, A. 2002. Infection of Arabidopsis with a necrotrophic pathogen, Botrytis cinerea, elicits various defense responses but does not induce systemic acquired resistance (SAR). Plant Mol. Biol. 48:267-276.

Guimaraes R. L., and Stotz, H. U. 2004. Oxalate production by Sclerotinia sclerotiorum deregulates guard cells during infection. Plant Physiol. 136:3703-3711.

Hu X., Bidney, D. L., Yalpani, N., Duvick, J. P., Crasta, O., Folkerts, O., and Lu, G. 2003. Overexpression of a gene encoding hydrogen peroxide-generating oxalate oxidase evokes defense responses in sunflower. Plant Physiol. 133:170-181.

Huh W. K., Falvo, J. V., Gerke, L. C., Carroll, A. S., Howson, R. W., Weissman, J. S., and O'Shea, E. K. 2003. Global analysis of protein localization in budding yeast. Nature 425:686-691.

Jordan D. B., Bacot, K. O., Carlson, T. J., Kessel, M., and Viitanen, P. V. 1999. Plant riboflavin biosynthesis. Cloning, chloroplast localization, expression, purification, and partial characterization of spinach lumazine synthase. J. Biol. Chem. 274:22114-22121.

Kesarwani M., Azam, M., Natarajan, K., Mehta, A., and Datta, A. 2000. Oxalate decarboxylase from Collybia velutipes: Molecular cloning and its overexpression to confer resistance to fungal infection in transgenic tobacco and tomato. J. Biol. Chem. 275:7230-7238.

Kolkman J. M., and Kelly, J. D. 2000. An indirect test using oxalate to determine physiological resistance to white mold in common bean. Crop Sci. 40:281-285.

Kolkman J. M., and Kelly, J. D. 2003. QTL conferring resistance and avoidance to white mold in common bean. Crop Sci. 43:539-548.

Lane B. G., Dunwell, J. M., Ray, J. A., Schmitt, M. R., and Cuming, A. C. 1993. Germin, a protein marker of early plant development, is an oxalate oxidase. J. Biol. Chem. 268:12239-12242. 
Laurie-Berry N., Joardar, V., Street, I. H., and Kunkel, B. N. 2006. The Arabidopsis thaliana JASMONATE INSENSITIVE 1 gene is required for suppression of salicylic acid-dependent defenses during infection by Pseudomonas syringae. Mol. Plant-Microbe Interact. 19:789-800.

Lawton K. A., Friedrich, L., Hunt, M., Weymann, K., Delaney, T., Kessmann, H., Staub, T., and Ryals, J. 1996. Benzothiadiazole induces disease resistance in Arabidopsis by activation of the systemic acquired resistance signal transduction pathway. Plant J. 10:71-82.

Li R., Rimmer, R., Buchwaldt, L., Sharpe, A. G., Seguin-Swartz, G., and Hegedus, D. D. 2004. Interaction of Sclerotinia sclerotiorum with Brassica napus: Cloning and characterization of endo- and exo-polygalacturonases expressed during saprophytic and parasitic modes. Fung. Genet. Biol. 41:754-765.

Lorenzo O., and Solano, R. 2005. Molecular players regulating the jasmonate signalling network. Curr. Opin. Plant Biol. 8:532-540.

Lorenzo O., Chico, J. M., Sanchez-Serrano, J. J., and Solano, R. 2004 JASMONATE-INSENSITIVE1 encodes a MYC transcription factor essential to discriminate between different jasmonate-regulated defense responses in Arabidopsis. Plant Cell 16:1938-1950.

Lumsden R. D. 1979. Histology and physiology of pathogenesis in plant diseases caused by Sclerotinia species. Phytopathology 69:890-896.

Mehta A., and Datta, A. 1991. Oxalate decarboxylase from Collybia velutipes. Purification, characterization, and cDNA cloning. J. Biol. Chem. 266:23548-23553.

Nickstadt A., Thomma, B. P. H. J., Feussner, I., Kangasjarvi, J., Zeier, J., Loeffler, C., Scheel, D., and Berger, S. 2004. The jasmonate-insensitive mutant jinl shows increased resistance to biotrophic as well as necrotrophic pathogens. Mol. Plant Pathol. 5:425-434.

Nürnberger T., and Lipka, V. 2005. Non-host resistance in plants: New insights into an old phenomenon. Mol. Plant Pathol. 6:335-345.

Persson K., Schneider, G., Jordan, D. B., Viitanen, P. V., and Sandalova, T. 1999. Crystal structure analysis of a pentameric fungal and an icosahedral plant lumazine synthase reveals the structural basis for differences in assembly. Prot. Sci. 8:2355-2365.

Pieterse C. M. J., Wees, S. C. M., van Pelt, J. A., Knoester, M., Laan, R., Gerrits, H., Weisbeek, P. J., and van Loon, L. C. 1998. A novel signalling pathway controlling induced systemic resistance in Arabidopsis. Plant Cell 10:1571-1580.

Purdy L. H. 1979. Sclerotinia sclerotiorum: History, diseases and symptomatology, host range, geographic distribution, and impact. Phytopathology 69:875-880.

Sambrook J., Fritsch, E. F., and Maniatis, T. 1989. Molecular Cloning: A Laboratory Manual, 2nd edition. Cold Spring Harbor Laboratory Press,
Cold Spring Harbor, NY, U.S.A.

Sang Y., Cui, D., and Wang, X. 2001. Phospholipase D and phosphatidic acid-mediated generation of superoxide in Arabidopsis. Plant Physiol. 126:1449-1458.

Shah J., Tsui, F., and Klessig, D. F. 1997. Characterization of a salicylic acid-insensitive mutant (sail) of Arabidopsis thaliana, identified in a selective screen utilizing the SA-inducible expression of the tms 2 gene. Mol. Plant-Microbe Interact. 10:69-78.

Shah J., Kachroo, P., and Klessig, D. F. 1999. The Arabidopsis ssil mutation restores pathogenesis-related gene expression in nprl plants and renders defensin gene expression salicylic acid dependent. Plant Cell 11:191-206.

Thomma B., Eggermont, K., Penninckx, I., Mauch-Mani, B., Vogelsang, R., Cammue, B., and Broekaert, W. 1998. Separate jasmonate-dependent and salicylate-dependent defense-response pathways in Arabidopsis are essential for resistance to distinct microbial pathogens. Proc. Natl. Acad. Sci. U.S.A. 95:15107-15111.

Thomma B., Eggermont, K., Tierens, K., and Broekaert, W. 1999. Requirement of functional ethylene-insensitive 2 gene for efficient resistance of Arabidopsis to infection by Botrytis cinerea. Plant Physiol. 121:1093-1102.

Thomma B. P. H. J., Penninckx, I. A. M. A., Broekaert, W. F., and Cammue, B. P. A. 2001. The complexity of disease signaling in Arabidopsis. Curr. Opin. Immunol. 13:63-68.

Thordal-Christensen H., Zhang, Z., Wei, Y., and Collinge, D. B. 1997. Subcellular localization of $\mathrm{H}_{2} \mathrm{O}_{2}$ in plants. $\mathrm{H}_{2} \mathrm{O}_{2}$ accumulation in papillae and hypersensitive response during the barley-powdery mildew interaction. Plant J. 11:1187-1194.

Ward E. R., Uknes, S. J., Williams, S. C., Dincher, S. S., Wiederhold, D. L., Alexander, D. C., Ahl-Goy, P., Metraux, J. P., and Ryals, J. A. 1991. Coordinate gene activity in response to agents that induce systemic acquired resistance. Plant Cell 3:1085-1094.

Xiao S., Dai, L., Liu, F., Wang, Z., Peng, W., and Xie, D. 2004. COS1: An Arabidopsis coronatine insensitive1 suppressor essential for regulation of jasmonate-mediated plant defense and senescence. Plant Cell 16:1132-1142.

Xie D.-X., Feys, B. F., James, S., Nieto-Rostro, M., and Turner, J. G. 1998. COI1: An Arabidopsis gene required for jasmonate-regulated defense and fertility. Science 280:1091-1094.

Xu L., Liu, F., Lechner, E., Genschik, P., Crosby, W. L., Ma, H., Peng, W. Huang, D., and Xie, D. 2002. The SCFCOI1 ubiquitin-ligase complexes are required for jasmonate response in Arabidopsis. Plant Cell 14:19191935. 